INTERNATIONAL JOURNAL OF ROBUST AND NONLINEAR CONTROL

Int. J. Robust Nonlinear Control 2009; 19:72-91

Published online 21 April 2008 in Wiley InterScience (www.interscience.wiley.com). DOI: 10.1002/rnc.1324

\title{
Feedback-feedforward individual pitch control for wind turbine load reduction
}

\author{
K. Selvam ${ }^{1, *, \dagger}$, S. Kanev ${ }^{2}$, J. W. van Wingerden ${ }^{3}$, T. van Engelen ${ }^{2}$ \\ and M. Verhaegen ${ }^{3}$ \\ ${ }^{1}$ Winwind Oy, Itälahdenkatu 15-17, FI-00210 Helsinki, Finland \\ ${ }^{2}$ Energy Research Center of the Netherlands, ECN Wind Energy, P.O. Box 1, 1755 ZG Petten, The Netherlands \\ ${ }^{3}$ Delft Center for Systems and Control, TU-Delft, Mekelweg 2, 2628 CD Delft, The Netherlands
}

\begin{abstract}
SUMMARY
This paper focuses on the problem of wind turbine fatigue load reduction by means of individual pitch control (IPC). The control approach has a two-degree-of-freedom structure, consisting of an optimal multivariable LQG controller and a feedforward disturbance rejection controller based on estimated wind speed signals. To make the control design problem time invariant, all signals are transformed to the non-rotating reference frame using the Coleman transformation. In the Coleman domain, the LQG control objective is minimization of the rotor tilt and yaw moments, whereas the feedforward controller tries to achieve even further improvement by rejecting the influence of the low-frequency components of the wind on the rotor moments. To this end, the tilt- and yaw-oriented components of the blade-effective wind speeds are approximated using stochastic random walk models, the states of which are then augmented with the turbine states and estimated using a Kalman filter. The effects of these (estimated) disturbances on the controlled outputs are then reduced using stable dynamic model inversion. The approach is tested and compared with the conventional IPC method in simulation studies with models of different complexities. The results demonstrate very good load reduction at not only low frequencies (1p blade fatigue load reduction) but also at the $3 \mathrm{p}$ frequency, giving rise to fatigue load reduction of the non-rotating turbine components. Copyright (C) 2008 John Wiley \& Sons, Ltd.
\end{abstract}

Received 26 July 2007; Revised 7 March 2008; Accepted 10 March 2008

KEY WORDS: wind turbine; fatigue load reduction; individual pitch control; LQG; wind estimation

\section{INTRODUCTION}

Wind turbine systems are considered as one of the most promising sources of renewable energy nowadays, with the installed capacity worldwide growing exponentially. Still, in order to reach the ambitious goals of the western governments, a significant reduction in the $\mathrm{kW} \mathrm{h}$ price is necessary,

*Correspondence to: K. Selvam, Winwind Oy, Itälahdenkatu 15-17, FI-00210 Helsinki, Finland.

†E-mail: kausihan.selvam@winwind.fi

Copyright (C) 2008 John Wiley \& Sons, Ltd. 
which necessitates even further upscaling of today's multi-MW wind turbines. These structures with increasing size are becoming more and more flexible, requiring an intelligent and robust control system that not only aims at good quality power control at the above-rated wind speeds but that primarily focuses on the active reduction of fatigue-relevant loads on the turbine components, as caused by wind shear, tower shadow, oblique inflow and turbulence.

At present, the majority of wind turbines are operating at variable rotor speed and use generator torque control and collective blade pitch control to produce as much power as possible at wind speeds below the rated speed, and to limit the captured power and the rotor speed at the above-rated wind speeds. The above-rated conventional power control strategy consists of two independent single-input single-output (SISO) control loops: generator torque control is used to achieve the desired static generator speed-torque relationship, and collective blade pitch angle (or speed) control consisting of simple PI (or PD) controller for keeping the generator speed at its rated value $[1-3]$.

Going one step further, this basic power regulation control can be extended by adding additional feedback loops with the objective of load reduction. For instance, additional generator torque control acting on the generator speed can be used to damp the drive train torsional vibration [1]. A tower top fore-aft acceleration measurement can be used by an additional collective pitch control loop to provide increased damping of the first tower fore-aft mode, whereas the sidewards tower acceleration can be fed back to a generator torque controller to achieve increased damping of the tower naying motion [4]. As all these (basic and additional) controllers influence each other, it is important to 'decouple' them by constraining their activities at different non-overlapping frequency ranges by means of proper filtering. To avoid such a disintegrated control design procedure, modern control design methods can be used for the design of multi-input multi-output (MIMO) controllers that directly aim at optimizing a trade-off between several design objectives at the same time, such as basic power regulation, robust stability to high-frequency unmodelled dynamics, improved resonant mode damping and control bandwidth limitation [5,6]. Such MIMO controllers, which are based on linearized turbine models, can be extended even further by making them adaptive to the constantly changing turbine's operating point (i.e. wind speed, rotor speed and pitch angle). This can be achieved by means of a linear parameter-varying (LPV) control design approach, as proposed in [7-11]. The price to pay for using such advanced optimal MIMO controllers is the increased computational complexity and the reduced transparency compared with conventional PID-based controllers.

Modern wind turbines offer the possibility of even more advanced load reductions by means of individual pitch control (IPC). To take advantage of that, additional measurements are needed, such as blade root bending moments. Since these are taken in the rotating reference frame, they give rise to a periodic system. This periodicity can fortunately be circumvented by transforming all quantities, defined on the rotating reference frame, to the fixed reference frame by using the so-called Coleman transformation [12], sometimes also referred to as $d-q$ transformation [13]. The blade root flapwise bending moments, for instance, are transformed into fictitious rotor tilt and yaw moments, which are used by the IPC to compute tilt-oriented and yaw-oriented pitch signals. These IPC controller outputs are transformed back to the original (rotating) reference frame, resulting in individual blade pitch signals. Besides the fact that the Coleman transformation makes the application of well-developed control theory for linear time-invariant (LTI) systems to the IPC problem possible, it also offers the additional advantage that it allows the collective pitch control design to be decoupled from the IPC design, since the IPC loop has only a negligible effect on the collective pitch control loop at low frequencies, where the controllers are active $[5,12]$. 
The reverse is, however, true to a lesser extent as discussed in Section 2.1, so that the IPC would best be designed for the turbine system with the basic collective pitch control in the loop, as done in this paper.

There are several IPC design methodologies suggested in the literature recently. In its conventional form, IPC is based on two separate SISO loops for rotor tilt and yaw moment reduction. Since the $1 \mathrm{p}$ (i.e. once per revolution) component of the blade loads is modulated by the Coleman transform into a static $(0 \mathrm{p})$ component in the rotor tilt and yaw moments, the conventional SISO loops use PI control laws to reduce the low-frequency content of the rotor moments $[13,14]$. This can further be improved by using optimal multivariable controllers, such as LQG [13-15] or $H_{\infty}$ control [5]. Although these methods result in a significant blade fatigue reduction, they offer little to no reduction on the fatigue loads of the non-rotating parts of the turbine, as those are dominated by the $3 p$ components of the loads in the fixed reference frame. To improve on that, the basic IPC control was extended in [12] to $2 p$ and $3 p$ (i.e. twice and three times per revolution) blade load reduction by means of modification of the Coleman transformation in such a way that, instead of the usual $1 \mathrm{p}$, now the $2 \mathrm{p}$ (respectively, $3 \mathrm{p}$ ) components in the blade root moments are transformed into 0p. Similar ideas are pursued in [16].

This paper describes a new IPC design method inspired by the optimal multivariable control in the Coleman domain $[13,15]$ on the one hand and by the wind speed estimation methods proposed in [17-19] on the other hand. In particular, the axial, tilt-oriented and yaw-oriented components of the blade-effective wind speed signals are modeled by stochastic random walk models, the states of which are then augmented with the states of the turbine to be estimated all together using a single Kalman filter. The wind signal estimates are subsequently used in a dynamic disturbance feedforward controller [20, Section 10.5.2]. The feedforward control action is added to the control action from an optimal feedback LQG controller. The resulting feedback-feedforward control structure is similar to the periodic disturbance accommodating control structure in [18], although the design approach proposed in this paper is fundamentally different in the following three aspects. First, the complete control design is now performed on an LTI system, as obtained by making use of the Coleman transform. This offers many possibilities for further improvements such as extension to LPV control for covering a wider range of operating points of the wind turbine, similar to the work of Bianchi for collective pitch control [7]. Secondly, the disturbance attenuation in [18] is based on a simple (static) pseudo-inverse of the system $B$ matrix, whereas the present approach focuses on a dynamic feedforward controller achieving minimization of the effect of the disturbance on the tilt and yaw moments. To this end, stable dynamic model inversion (SDMI) is used [21]. And third, the current method deals with blade-effective wind speeds, which allows for more accurate approximation of the loads on the individual blades than when only rotor-effective wind speed is considered, as in [18].

The method is tested in simulation on two models of different complexities. First, a simple rigid turbine model with just a few degrees of freedom is used, which provides useful insights and serves as a good basis for the analysis of the presented control strategy. Next, a detailed aero-elastic linear model, generated by the computer program Turbu [22], is used for more detailed study of the control strategy. The results are compared with the results obtained with a conventional PI-based IPC. It is demonstrated that the present method achieves a significant improvement over the conventional one in that it reduces the rotor tilt and yaw moments over a much larger frequency band, including the fatigue-relevant $3 p$ components.

The paper is organized as follows. In the following section the models used for the IPC design are introduced. Section 3 presents the proposed feedback-feedforward method for IPC, which is 
subsequently tested and compared with conventional IPC in simulations in Section 4. The paper concludes with some final remarks in Section 5.

\section{WIND TURBINE MODEL}

For IPC synthesis and analysis for load reduction, it is useful to have simple LTI turbine models that contain enough detail about the system loads. To this end it is necessary to get rid of the azimuth dependence of some signals, which can be achieved by applying a multi-blade coordinate transformation (Coleman transformation [23]) on the periodic wind turbine model. One way to obtain such detailed linearized turbine models is by using TURBU [22], ECN's linear analysis and simulation code for horizontal axis wind turbines. However, since the state-space model generated by TURBU is usually of a rather high-order model and is, hence, less transparent, a simple firstprinciples rigid turbine model is first introduced to provide the necessary insight and to outline the basic principles involved in the generation of a TURBU model.

\subsection{Simple wind turbine model}

The simple linear model of a three-bladed turbine, taken from [12], has the following features:

(i) individually pitch-controlled rigid blades;

(ii) main rotation and rigid drive train;

(iii) first fore-aft and sidewards tower bending mode;

(iv) controllable generator torque.

The model is excited by realistic blade-effective wind speed signals, accounting for $k p$-effects on the rotor blades, $k=1,2, \ldots$, due to rotational wind field sampling, tower shadow and wind shear. Stationary aerodynamic conversion is utilized for the derivation of blade root bending moments. A schematic layout of the wind turbine model is depicted in Figure 1. How this model is derived is summarized below.

2.1.1. Linearized aerodynamic conversion. Linear blade element momentum (BEM) theory is used for computing linearized aerodynamics. To keep the model as simple as possible, unsteady aerodynamics and wake effects have not been taken into account. The BEM aerodynamics is based on a linear static map that relates the flapwise wind speeds $v_{\mathrm{ff}_{i}}, i=1,2,3$, to flapwise and leadwise blade root bending moments and forces. The flapwise relative wind speed variation $v_{\mathrm{fl}_{i}}$ is defined as the sum of the blade-effective wind speed $u_{i}$ and the upwind motion of the rotor blade. The latter is caused by fore-aft tower bending only since rigid blades are assumed. The upwind structural motion involves both the fore-aft translation $x_{\mathrm{fa}}$ and tilt rotation $\dot{\phi}_{\mathrm{fa}}$ of the tower top. The latter has an azimuth-dependent effect on the relative wind speed, which varies over the rotor radius. It is assumed that wind acts at one single point of the blade, located at a distance of $\frac{3}{4}$ of the blade radius $R_{\mathrm{b}}$. Hence, denoting $H$ as the tower height, and assuming that the fore-aft tower motion can be approximated by the motion of a prismatic beam, for which it is known that the ratio between rotation and displacement is given by $3 / 2 \mathrm{H}$, the flapwise relative wind speed $v_{\mathrm{fl}_{i}}$ can be expressed as follows:

$$
v_{\mathrm{fl}_{i}}=u_{i}-\dot{x}_{\mathrm{fa}}+\sin \left(\psi_{i}\right) \frac{3}{2 H} \frac{3 R_{\mathrm{b}}}{4} \dot{x}_{\mathrm{fa}}
$$




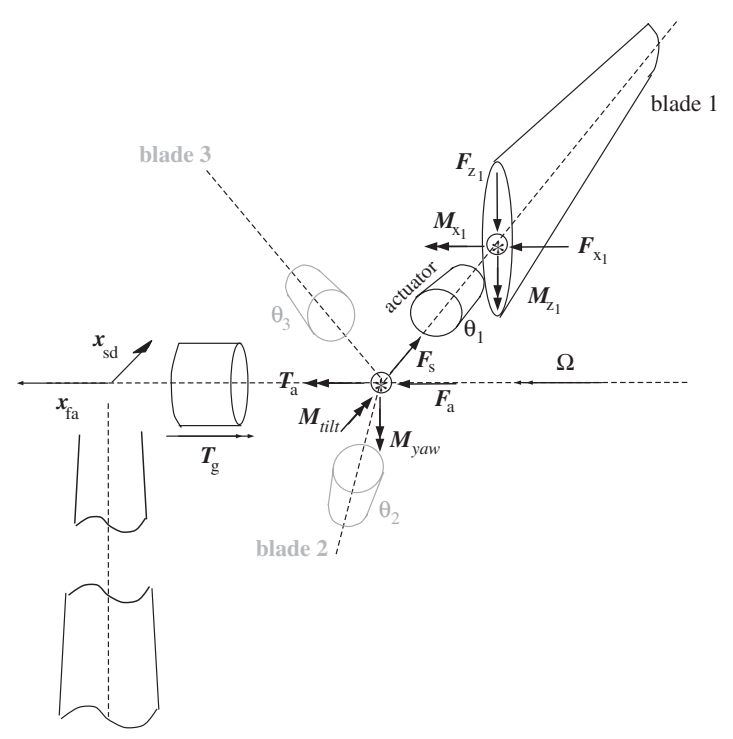

Figure 1. A schematic layout of the simplified wind turbine model.

where $\psi_{i}$ is the azimuth angle of blade $i$, measured with respect to the $y$-axis of the fixed reference frame. The rotor azimuth angle $\psi$ is defined as the azimuth angle of the first blade, i.e. $\psi=\psi_{1}$.

With this notation, for the $i$ th blade, the variations of these aerodynamic moments and forces are given by

$$
\left[\begin{array}{c}
M_{z, i} \\
F_{x, i} \\
M_{x, i} \\
F_{z, i}
\end{array}\right]=\left[\begin{array}{ll}
h_{M_{z}} & k_{M_{z}} \\
h_{F_{x}} & k_{F_{x}} \\
h_{M_{x}} & k_{M_{x}} \\
h_{F_{z}} & k_{F_{z}}
\end{array}\right]\left[\begin{array}{c}
v_{\mathrm{fl}_{i}} \\
\theta_{i}
\end{array}\right]
$$

where $\theta_{i}$ are the pitch angle variations, $M_{z, i}$ and $M_{x, i}$ are the variations in the flapwise and the leadwise blade root bending moments and $F_{x, i}$ and $F_{z, i}$ are the flapwise and the leadwise forces. The gains $h_{M_{z}} \cdots k_{F_{z}}$ are derived from the power and thrust coefficient data in a chosen working point, characterized by wind speed, rotor speed and pitch angle. The derivation is constrained by the assumption of equal aerodynamic efficiency along the blade radius, which implies a linear increasing flapwise force per unit span over the rotor radius and constant leadwise force per unit span, see [4].

The variations in aerodynamic torque $T_{\mathrm{a}}$, axial force $F_{\mathrm{a}}$, rotor tilt moment $M_{\mathrm{tilt}}$ and sidewards force at the tower top $F_{\mathrm{s}}$ are then given by

$$
\left[\begin{array}{c}
T_{\mathrm{a}} \\
F_{\mathrm{a}} \\
M_{\mathrm{tilt}} \\
F_{\mathrm{s}}
\end{array}\right]=\sum_{i=1}^{3}\left[\begin{array}{c}
M_{x, i} \\
F_{x, i} \\
-M_{z, i} \sin \psi_{i} \\
-F_{z, i} \sin \psi_{i}
\end{array}\right]
$$


2.1.2. Periodic linear model equations. The drive train is accelerated by the aerodynamic driving torque $T_{\mathrm{a}}$ and decelerated by the generator torque $T_{\mathrm{g}}$. Under the assumption of stiff drive train, the following equation holds for the rotor speed $\Omega$ (generator torque is transformed to slow shaft equivalent):

$$
J \dot{\Omega}=T_{\mathrm{a}}-T_{\mathrm{g}}
$$

where $J$ is the total inertia (rotor and generator). By noting that $\sum_{i=1}^{3} \sin \psi_{i}=0$ and $\sum_{i=1}^{3} \sin ^{2} \psi_{i}=$ $\frac{3}{2}$, the following expression for the linearized torques and moments can be derived from equations (1)-(3):

$$
\left[\begin{array}{c}
T_{\mathrm{a}} \\
F_{\mathrm{a}} \\
M_{\text {tilt }} \\
F_{\mathrm{s}}
\end{array}\right]=\sum_{i=1}^{3}\left[\begin{array}{ccc}
h_{M_{x}} & k_{M_{x}} & -h_{M_{x}} \\
h_{F_{x}} & k_{F_{x}} & -h_{F_{x}} \\
-\sin \psi_{i} h_{M_{z}} & -\sin \psi_{i} k_{M_{z}} & -\frac{9 R_{\mathrm{b}}}{16 H} h_{M_{z}} \\
-\sin \psi_{i} h_{F_{z}} & -\sin \psi_{i} k_{F_{z}} & -\frac{9 R_{\mathrm{b}}}{16 H} h_{F_{z}}
\end{array}\right]\left[\begin{array}{c}
u_{i} \\
\theta_{i} \\
\dot{x}_{\mathrm{fa}}
\end{array}\right]
$$

The variables of the included tower model are the fore-aft $x_{\mathrm{fa}}$ and the sidewards $x_{\mathrm{sd}}$ displacements, respectively. The fore-aft tower motion is driven by the aerodynamic thrust force $F_{\mathrm{a}}$ and the tilt moment $M_{\text {tilt }}$, whereas the sidewards motion is driven by the generator torque $T_{\mathrm{g}}$ and the sidewards aerodynamic force $F_{\mathrm{s}}$. Only the first bending modes of the tower in the fore-aft and sidewards directions are considered. These are approximated by the following second-order differential equations:

$$
\begin{aligned}
& m_{\mathrm{tw}} \ddot{x}_{\mathrm{fa}}+s_{\mathrm{tw}} x_{\mathrm{fa}}+d_{\mathrm{tw}} \dot{x}_{\mathrm{fa}}=F_{\mathrm{a}}-\frac{3}{2 H} M_{\mathrm{tilt}} \\
& m_{\mathrm{tw}} \ddot{x}_{\mathrm{sd}}+s_{\mathrm{tw}} x_{\mathrm{sd}}+d_{\mathrm{tw}} \dot{x}_{\mathrm{sd}}=\frac{3}{2 H} T_{\mathrm{g}}+F_{\mathrm{s}}
\end{aligned}
$$

where $m_{\mathrm{tw}}, s_{\mathrm{tw}}$ and $d_{\mathrm{tw}}$ are tower top equivalent tower mass, stiffness coefficient and damping coefficient, respectively. Their numerical values can be derived from the following structural data:

- the axial tower displacement at unity force;

- damping rate of the first tower bending mode;

- average of the first fore-aft and sidewards frequencies.

Again, the multiplication factor $3 / 2 \mathrm{H}$ in Equation (6) is derived based on the prismatic beam approximation of the tower, as discussed above.

Finally, substitution of the expressions in Equation (5) into (4) and (6) results in

$$
\begin{aligned}
J \dot{\Omega}= & h_{M_{z}} \sum_{i=1}^{3} u_{i}+k_{M_{x}} \sum_{i=1}^{3} \theta_{i}-3 h_{M_{x}} \dot{x}_{\mathrm{fa}}-T_{\mathrm{g}} \\
m_{\mathrm{tw}} \ddot{x}_{\mathrm{fa}}= & \sum_{i=1}^{3}\left(h_{F_{x}}+\frac{3}{2 H} \sin \psi_{i} h_{M_{z}}\right) u_{i}+\sum_{i=1}^{3}\left(k_{F_{x}}+\frac{3}{2 H} \sin \psi_{i} k_{M_{z}}\right) \theta_{i} \\
& +\left(\frac{81 R_{\mathrm{b}}}{32 H^{2}} h_{M_{z}}-3 h_{F_{x}}-d_{\mathrm{tw}}\right) \dot{x}_{\mathrm{fa}}-s_{\mathrm{tw}} x_{\mathrm{fa}}
\end{aligned}
$$




$$
m_{\mathrm{tw}} \ddot{x}_{\mathrm{sd}}=-h_{F_{z}} \sum_{i=1}^{3} \sin \psi_{i} u_{i}-k_{F_{z}} \sum_{i=1}^{3} \sin \psi_{i} \theta_{i}-\frac{27 R_{\mathrm{b}}}{16 H} h_{F_{z}} \dot{x}_{\mathrm{fa}}+\frac{3}{2 H} T_{\mathrm{g}}-s_{\mathrm{tw}} x_{\mathrm{sd}}-d_{\mathrm{tw}} \dot{x}_{\mathrm{sd}}
$$

It is assumed in this paper that besides the generator speed, the three blade flapwise bending moments are measured. Hence, these are included as output equations in the model

$$
M_{z_{i}}=h_{M_{z}}\left(\sin \psi_{i} \frac{9 R_{\mathrm{b}}}{8 H}-1\right) \dot{x}_{\mathrm{fa}}+h_{M_{z}} u_{i}+k_{M_{z}} \theta_{i}, \quad i=1,2,3
$$

2.1.3. The Coleman transformation. Equation (7) contains coefficients that depend on the blade azimuth angles, giving rise to a time-varying system. However, these equations can be transformed to a purely time-invariant linear system by means of formulating the structural and aerodynamic degrees of freedom for the rotor in multi-blade coordinates using the Coleman transform [23]. The Coleman transformation, also called multi-blade transformation, maps the individual blade coordinates present in the periodic frame of reference into a fixed frame of reference. In other words, the Coleman transformation maps the blade coordinates to the tower coordinates. In due process the periodic terms of the aero-elastic equations will be eliminated, as the number of blades is three, meaning the rotor is isotropic (identical and symmetrically mounted blades) and the inflow to the rotor is uniform.

The periodic terms are eliminated by multi-blade transformation, because the coordinates of the model are defined in the same frame of reference as explained in [24]. For example, the physical coordinate $q_{i}$ for blade $i$ of a three-bladed wind turbine in the rotating frame is represented as follows:

$$
q_{i}(t)=q_{1}^{\mathrm{cm}}(t)+q_{2}^{\mathrm{cm}}(t) \sin \left(\psi t+\frac{2 \pi}{3}(i-1)\right)+q_{3}^{\mathrm{cm}}(t) \cos \left(\psi t+\frac{2 \pi}{3}(i-1)\right)
$$

The three multi-blade coordinates $q_{i}^{\mathrm{cm}}, i=1,2,3$, replace the blade coordinates $q_{i}, i=1,2,3$. In this manner, the blade pitch angles and the blade-effective wind speeds can be represented as

$$
\left[\begin{array}{l}
\theta_{1}(t) \\
\theta_{2}(t) \\
\theta_{3}(t)
\end{array}\right]=\mathbf{P}\left[\begin{array}{l}
\theta_{1}^{\mathrm{cm}}(t) \\
\theta_{2}^{\mathrm{cm}}(t) \\
\theta_{3}^{\mathrm{cm}}(t)
\end{array}\right],\left[\begin{array}{l}
u_{1}(t) \\
u_{2}(t) \\
u_{3}(t)
\end{array}\right]=\mathbf{P}\left[\begin{array}{l}
u_{1}^{\mathrm{cm}}(t) \\
u_{2}^{\mathrm{cm}}(t) \\
u_{3}^{\mathrm{cm}}(t)
\end{array}\right]
$$

where the matrix

$$
\mathbf{P}=\left[\begin{array}{ccc}
1 & \sin \psi_{1}(t) & \cos \psi_{1}(t) \\
1 & \sin \psi_{2}(t) & \cos \psi_{2}(t) \\
1 & \sin \psi_{3}(t) & \cos \psi_{3}(t)
\end{array}\right]
$$

is referred to as the Coleman transformation matrix. Its inverse

$$
\mathbf{P}^{-1}=\left[\begin{array}{ccc}
\frac{1}{3} & \frac{1}{3} & \frac{1}{3} \\
\frac{2}{3} \sin \psi_{1}(t) & \frac{2}{3} \sin \psi_{2}(t) & \frac{2}{3} \sin \psi_{3}(t) \\
\frac{2}{3} \cos \psi_{1}(t) & \frac{2}{3} \cos \psi_{2}(t) & \frac{2}{3} \cos \psi_{3}(t)
\end{array}\right]
$$


is used to convert the multi-blade coordinates back to the rotating reference frame. For instance, given the multi-blade coordinates of the blade flapwise root bending moments $M_{z_{i}}^{\mathrm{cm}}, i=1,2,3$, the following can be expressed:

$$
\left[\begin{array}{l}
M_{z_{1}}^{\mathrm{cm}}(t) \\
M_{z_{2}}^{\mathrm{cm}}(t) \\
M_{z_{3}}^{\mathrm{cm}}(t)
\end{array}\right]=\mathbf{P}^{-1}\left[\begin{array}{l}
M_{z_{1}}(t) \\
M_{z_{2}}(t) \\
M_{z_{3}}(t)
\end{array}\right]
$$

The second and the third multi-blade flap moment coordinates $M_{z_{3}}^{\mathrm{cm}}$ and $M_{z_{3}}^{\mathrm{cm}}$ have tilt and yaw orientation. In fact, neglecting the effects of shearing and tensile forces, and pitchwise moments by the blades on the hub center, these two multi-blade moments are proportional to the tilt $\left(M_{\text {tilt }}\right)$ and yaw $\left(M_{\text {yaw }}\right)$ moments at the hub center [4]:

$$
\left[\begin{array}{c}
M_{\text {tilt }} \\
M_{\text {yaw }}
\end{array}\right]=\frac{3}{2}\left[\begin{array}{c}
-M_{z_{2}}^{\mathrm{cm}} \\
M_{z_{3}}^{\mathrm{cm}}
\end{array}\right]
$$

The first multi-blade flapwise moment $M_{1}^{\mathrm{cm}}$ only has a physical interpretation in terms of hub loading, and is not commonly used by IPC algorithms.

2.1.4. The LTI model. Although the equations of motion depend on the azimuth angle $\psi(t)$, they do not include any state variables related to the rotating rotor blade and rotor shaft except for the rotational speed $\Omega$. Since $\Omega$ has a co-axial orientation, no state variable needs to be transformed. The flapwise blade root bending moments, the pitch angles and the blade-effective wind speeds, on the other hand, are variables that do need to be transformed to arrive at an LTI model. Indeed, it can easily be verified that using Equations (9)-(11), the time-varying model (7)-(8) takes the following form:

$$
\begin{aligned}
J \dot{\Omega}= & -3 h_{M_{x}} \dot{x}_{\mathrm{fa}}+3 k_{M_{x}} \theta_{1}^{\mathrm{cm}}+3 h_{M_{x}} u_{1}^{\mathrm{cm}}-T_{\mathrm{g}} \\
m_{\mathrm{tw}} \ddot{x}_{\mathrm{fa}}= & -s_{\mathrm{tw}} x_{\mathrm{fa}}-\left(d_{\mathrm{tw}}+3 h_{F_{x}}-\frac{81 R_{\mathrm{b}}}{32 H^{2}} h_{M_{z}}\right) \dot{x}_{\mathrm{fa}}+3 k_{F_{x}} \theta_{1}^{\mathrm{cm}} \\
& +\frac{9}{4 H} k_{M_{z}} \theta_{2}^{\mathrm{cm}}+3 h_{F_{x}} u_{1}^{\mathrm{cm}}+\frac{9}{4 H} h_{M_{z}} u_{2}^{\mathrm{cm}} \\
m_{\mathrm{tw}} \ddot{x}_{\mathrm{sd}}= & \frac{3}{2 H} T_{\mathrm{g}}-\frac{27 R_{\mathrm{b}}}{16 H} h_{F_{z}} \dot{x}_{\mathrm{fa}}-\frac{3}{2} k_{F_{z}} \theta_{2}^{\mathrm{cm}}-\frac{3}{2} h_{F_{z}} u_{2}^{\mathrm{cm}}-s_{\mathrm{tw}} x_{\mathrm{sd}}-d_{\mathrm{tw}} \dot{x}_{\mathrm{sd}} \\
M_{z_{1}}^{\mathrm{cm}}= & -h_{M_{z}} \dot{x}_{\mathrm{fa}}+k_{M_{z}} \theta_{1}^{\mathrm{cm}}+h_{M_{z}} u_{1}^{\mathrm{cm}} \\
M_{\mathrm{tilt}}= & -\frac{27 R_{\mathrm{b}}}{16 H} h_{M_{z}} \dot{x}_{\mathrm{fa}}-\frac{3}{2} k_{M_{z}} \theta_{2}^{\mathrm{cm}}-\frac{3}{2} h_{M_{z}} u_{2}^{\mathrm{cm}} \\
M_{\text {yaw }}= & \frac{3}{2} k_{M_{z}} \theta_{3}^{\mathrm{cm}}+\frac{3}{2} h_{M_{z}} u_{3}^{\mathrm{cm}}
\end{aligned}
$$

where in the derivation of the last equation the equality $\sum_{i=1}^{3} \sin \psi_{i} \cos \psi_{i}=0$ has been used. Clearly, these equations represent an LTI system. Figure 2 gives a block-schematic representation of the resulting LTI system. Note that apart from the rotor speed $\Omega$ and the generator torque $T_{\mathrm{g}}$, 


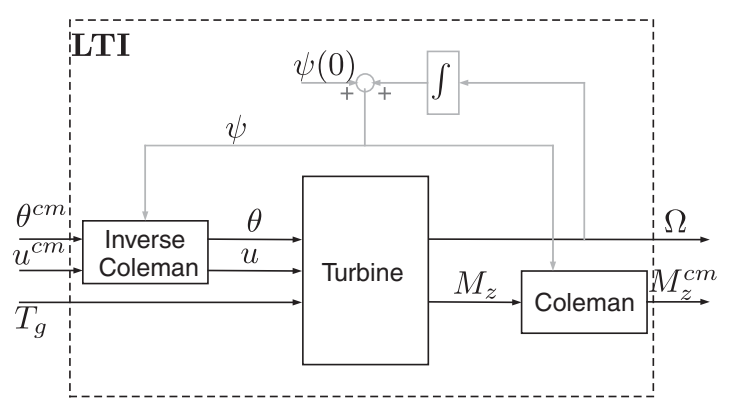

Figure 2. Conversion of the periodic model into a linear model using the Coleman transformation.

the inputs and outputs of the model are in multi-blade coordinates, and are hence only fictitious. Therefore, if an LTI controller is designed based on this system representation, then when implementing it with the true turbine it is necessary to convert its inputs and outputs back to the original rotating reference frame. In other words, the blocks 'Coleman' and 'inverse Coleman' in Figure 2 will at the end be included into the controller, which will actually make the final controller time varying.

The conventional IPC methods consist of two individual SISO (usually of PI structure) pitch controllers, one from $M_{\text {tilt }}$ to $\theta_{2}^{\mathrm{cm}}$, and the other one from $M_{\text {yaw }}$ to $\theta_{3}^{\mathrm{cm}}$. The output $M_{z_{1}}^{\mathrm{cm}}$ is not used. These IPC controllers are designed separately from the collective pitch controller that has the rotor speed $\Omega$ as input and $\theta_{1}^{\mathrm{cm}}$ as output. Clearly, the yaw-oriented channel (18) is (in this simple model) completely decoupled from the other channels. This, however, is not true for the tilt-oriented moment $M_{\text {tilt }}$ as it is influenced by the collective control loop via the tower fore-aft speed $\dot{x}_{\mathrm{fa}}$. Therefore, the design of the tilt moment control loop should be performed keeping in mind the presence of the collective pitch controller. The latter, in turn, can be designed separately from both IPCs as the rotor speed is not influenced by the individual pitch actions $\theta_{2}^{\mathrm{cm}}$ and $\theta_{3}^{\mathrm{cm}}$.

Note also that the sidewards tower motion does not have any affect on the control loops as it does not influence either the rotor speed or the tilt and yaw moments, i.e. these dynamics are unobservable. Hence, Equation (15) can be removed from this simple model.

\subsection{Detailed TURBU model}

The ECN computer code TURBU [22] generates elaborate linearized aero-elastic models of threebladed horizontal axis wind turbines. These models include considerable features that are necessary for control design and aero-elastic stability analysis, such as bending and torsion deformation, (unsteady) aerodynamic and hydrodynamic conversions and wake dynamics. All model inputs for the drive train and rotor blades are transformed into multi-blade coordinates before they enter the LTI model, and the model outputs from the drive train and rotor blades are transformed back to rotating coordinates. A linear model is computed for a given aerodynamic equilibrium state. The latter is derived via the BEM theory. The average deformation state is matched to the aerodynamic equilibrium. This is based on non-linear propagation of the deformation of the individual elements caused by the average loading. The average deformation per element is based on slender beam bending theory. A multi-body approach is used to model the structural dynamics, as illustrated in [22]. The multi-body wind turbine model has $N$ elements per blade $\left(\left\{D_{i}, E_{i}, F_{i}\right\}, i=1,2, \ldots, N\right)$ 
and $M$ elements for the tower $\left(S_{i}, i=1,2, \ldots, M\right)$, with each element having 5 degrees of freedom. There are 6 degrees of freedom in the rotor shaft $R_{\mathrm{r}}$.

A typical TURBU model (with $N=14$ and $M=15$ ) has around 600 state variables, and therefore needs simplification when used for control design. For reducing the model order, TURBU provides model reduction based on the elimination of high-frequency modes. This allows significant model order reduction without any loss of accuracy in the dynamic behavior of the lower-frequency modes that are within the bandwidth of the controllers. This yields a model order of about 150 . This reduced-order model is here referred to as the multi-body model. In addition to that, TURBU allows for modeling only a distinct number of degrees of freedom in the blade roots and tower bottom. The model obtained in this manner is referred to as the lumped model. It has only 28 states and still models dynamic pitch servo actuation. Figure 3 shows the frequency responses from the multi-blade pitch angle signals $\theta_{i}^{\mathrm{cm}}, i=1,2,3$, to the rotor speed $\Omega$, tilt moment $M_{\text {tilt }}$ and yaw moment $M_{\text {yaw }}$ for both the full multi-body (solid line) and reduced lumped (dashed line) models. Sufficient accuracy is observed at frequencies below $1 \mathrm{~Hz}$.

From the frequency plots it is clear that at low frequencies (below about $0.3 \mathrm{~Hz}$ ) there is little coupling between the channel $\theta_{1}^{\mathrm{cm}} \rightarrow \Omega$ on the one hand and the IPC channels $\theta_{2}^{\mathrm{cm}} \rightarrow M_{\mathrm{tilt}}$ and $\theta_{3}^{\mathrm{cm}} \rightarrow M_{\text {yaw }}$ on the other hand. However, above this frequency there is a clear interaction between these loops. Therefore, when the collective controller is separately designed from the IPC it is very important that these loops be further 'decoupled' using suitable filters. It is especially important that structural resonant frequencies that are well inside the bandwidth of the collective controller (e.g.
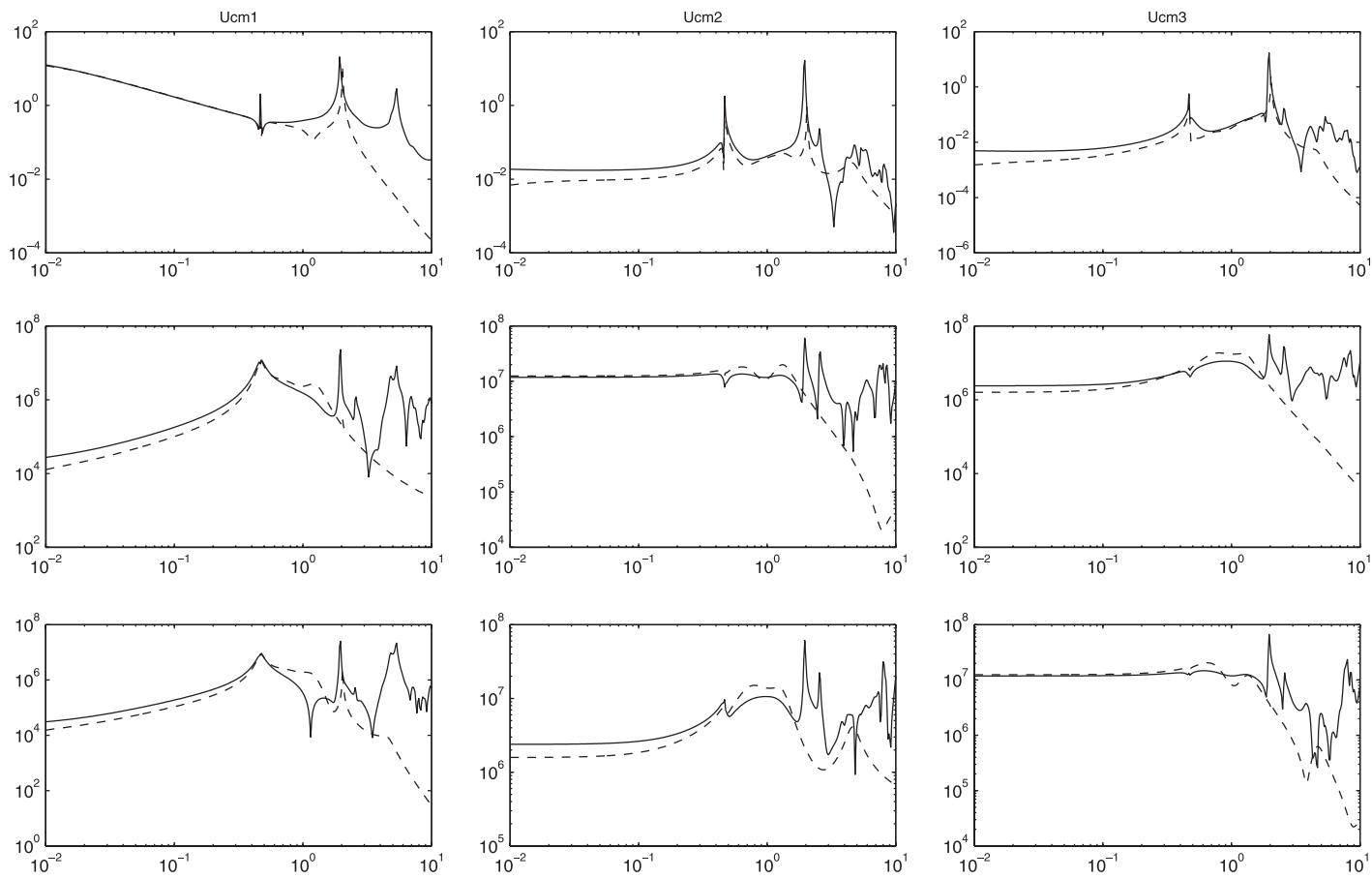

Figure 3. Frequency response from multi-blade pitch angles to rotor speed, tilt moment and yaw moment, for both the multi-body (solid) and the lumped models (dashed). 
the tower frequency at about $0.47 \mathrm{~Hz}$ ) are filtered out from $\theta_{1}^{\mathrm{cm}}$ so that it does not get influenced by the IPC control actions.

Similar conclusions can be made for the IPC loops, which are only decoupled from the collective loop at low frequencies, and if designed independently, filtering should be used to reduce highfrequency effects from the collective pitch action. However, in this paper a different approach is used for the IPC design, which does not need additional filter design. The starting point is the design of the collective pitch controller and the generator torque controller using conventional methods, including filters, as discussed above. These controllers are then interconnected with the turbine, so that the IPC design is performed on the closed-loop turbine system with the generator torque control and the collective pitch control in the loop. Then, a multivariable robust control design approach is used to synthesize one MIMO IPC loop that stabilizes the turbine and minimizes a suitably defined performance criterion based on the rotor moments. Of course, one could choose to include also the collective pitch and generator torque control loops into the MIMO controller; this is not done here as the present approach assumes that the speed and power loops are given, and it focuses on the inclusion of additional actuation through IPC for the purpose of fatigue load reduction. Note that this MIMO approach also takes into account the coupling between the tiltand the yaw-oriented multi-blade coordinates, whose coupling is clearly much stronger than the coupling with the collective pitch loop (see Figure 3). This coupling was absent in the simple model from the previous subsection.

\section{CONTROLLER DESIGN}

In this section the proposed IPC design approach is explained. Since it is compared in the following section with the conventional SISO approach to IPC, it is briefly described next.

\subsection{Conventional SISO control approach}

The conventional IPC is a scalar approach based on the assumption that the multi-blade coordinate transformation, described in Section 2.1.3, results in three independent time-invariant control loops: from the collective pitch angle to the rotor speed, from the yaw-oriented pitch angle to the yaw moment and from the tilt-oriented pitch angle to the tilt angle. It is assumed that the generator speed is measured.

The speed regulation loop is usually based on a PI compensator that has as input the difference between the filtered generator speed and its rated value. The filter, used in the simulations, includes

- A low-pass filter (inverse Chebychev [type II], fourth order, $20 \mathrm{~dB}$ reduction) with a cut-off frequency of $(3 p-0.8) \mathrm{rad} / \mathrm{s}$.

- A notch filter (elliptic filter, fourth order, $30 \mathrm{~dB}$ reduction) with stop band $\left[0.8 \omega_{\mathrm{tsd}}, 1.05 \omega_{\mathrm{tsd}}\right]$, where $\omega_{\text {tsd }}$ is the first tower naying frequency.

- A notch filter (elliptic filter, second order, $30 \mathrm{~dB}$ reduction) with stop band [0.8 $\left.\omega_{\mathrm{cll}}, 1.05 \omega_{\mathrm{cll}}\right]$, where $\omega_{\text {cll }}$ is the collective lead-lag frequency of the blades.

The PI compensator is designed to achieve a gain margin of 2 and a phase margin of $45^{\circ}$.

The torque controller is designed for achieving constant power production by keeping the generator torque equal to the rated power $P_{\mathrm{r}}$ divided by the rotor speed. After linearization around the rated generator speed $\Omega_{\mathrm{g}, \mathrm{r}}$, the generator controller has the form of a P-compensator with gain 
$\left(-P_{\mathrm{r}} / n_{\mathrm{gb}} \Omega_{\mathrm{g}, \mathrm{r}}^{2}\right)$, where $n_{\mathrm{gb}}$ is the gearbox ratio. It should be noted that this generator controller has a slightly destabilizing effect on the rotor speed as the generator torque decreases when the generator speed increases.

The load regulation loops aim at blade fatigue load reduction by reducing the $1 \mathrm{p}$ load component in the blade root bending moments. It is demonstrated in [12] that $(3 m \pm 1)$ components, $m=$ $1,2, \ldots$, in the load spectrum of the blade-effective wind speed signals $u_{i}, i=1,2,3$, contribute to the $3 m$ components in the multi-blade wind coordinates $u_{i}^{\mathrm{cm}}$. In other words, $1 \mathrm{p}$ loads on the blades are modulated into $0 \mathrm{p}$ loads on the tilt and yaw moments, meaning that the former can be reduced by reducing the static loads on the rotor moments. This can be achieved by means of a simple I-compensator. In order to prevent the influence of higher harmonics it is necessary to apply low-pass filtering around and beyond $3 \mathrm{p}$.

\subsection{Feedback-feedforward multivariable control approach to IPC}

In this section the multivariable control approach to IPC, proposed in this paper, will be introduced. It has a two-degree-of-freedom structure, containing an optimal feedback LQG controller from the rotor tilt and yaw moments to the multi-blade pitch angles $\theta_{i}^{\mathrm{cm}}$, and a feedforward disturbance rejection controller acting on the estimated multi-blade wind signals and producing additional multi-blade pitch angles.

As discussed above, this paper assumes that the collective pitch controller $C_{\mathrm{col}}(s)$ and generator torque controller $C_{\text {gen }}(s)$ (including the filters) are available and interconnected with the wind turbine to regulate the produced power by controlling the generator torque and the rotor speed, as explained in the previous subsection. Below it is assumed that the controllers $C_{\text {col }}(s)$ and $C_{\text {gen }}(s)$ are interconnected to the linear turbine model, be it the simple rigid model (12)-(15) or the multibody TURBU model (by substituting $\theta_{1}^{\mathrm{cm}}=C_{\mathrm{col}}(s) \Omega$ and $T_{\mathrm{g}}=C_{\mathrm{gen}} \Omega$ in the turbine model), after which discretization is performed, resulting in

$$
\begin{aligned}
x_{\mathrm{wt}}(k+1) & =A_{\mathrm{wt}} x_{\mathrm{wt}}(k)+B_{\mathrm{wt}, \theta} \theta_{23}^{\mathrm{cm}}(k)+B_{\mathrm{wt}, u} u^{\mathrm{cm}}(k) \\
y(k) & =C_{\mathrm{wt}} x_{\mathrm{wt}}(k)+D_{\mathrm{wt}, \theta} \theta_{23}^{\mathrm{cm}}(k)+D_{\mathrm{wt}, u} u^{\mathrm{cm}}(k)+v(k)
\end{aligned}
$$

where the state $x_{\mathrm{wt}}$ contains the state of the wind turbine model and the states of the torque and pitch controllers, $\theta_{23}^{\mathrm{cm}}=\left[\theta_{2}^{\mathrm{cm}}, \theta_{3}^{\mathrm{cm}}\right]^{\mathrm{T}}$ is the input, $u^{\mathrm{cm}}=\left[u_{1}^{\mathrm{cm}}, u_{2}^{\mathrm{cm}}, u_{3}^{\mathrm{cm}}\right]^{\mathrm{T}}$ is the disturbance input, $y=\left[M_{\mathrm{tilt}}, M_{\mathrm{yaw}}\right]$ is the measured output and $v \in \mathbb{R}^{2}$ is a zero-mean white noise process with covariance matrix $Q_{v}=Q_{v}^{\mathrm{T}}>0$. Note that the rotor tilt and yaw moments are assumed to be measured. In practice, conventional wire strain gauges can be used to measure the flapwise bending moments at the blade roots, which can then be converted into $M_{\text {tilt }}$ and $M_{\text {yaw }}$ using the Coleman transformation. Although strain gauges are not very reliable devices as such for this application, due to the potential danger caused by lighting, the recent developments of optical strain gages are likely to overcome this disadvantage.

3.2.1. Design of the optimal LQG controller. The optimal LQG controller consists of a linear quadratic regulator (LQR) and a Kalman filter. However, the conventional assumption in the Kalman filter design that the external input is a random white Gaussian process is clearly not satisfied for model (19), as the multi-blade wind signals $u^{\mathrm{cm}}(k)$ have no flat spectrum. To circumvent this problem, one can identify a stochastic linear model $M_{\text {wind }}(z)$ that has (approximately) the same spectrum as the wind signals $u^{\mathrm{cm}}(k)$. This would allow modeling of $u^{\mathrm{cm}}$ as the output of a filtered 
white noise process, $u^{\mathrm{cm}}=M_{\text {wind }}(z) w$. Substituting this into the turbine model (19), and augmenting the states $x^{w t}$ with the filter model states, will yield a new model that has the white noise process $w$ as external input, so that standard Kalman filter design can be performed. This approach, however, requires (a) that the spectrum of the multi-blade wind signals is given and (b) that it is accurately represented by a linear model of low order (in order to keep the order of the final controller low). To avoid this approach, one might instead use a much simpler wind modeling, by noting that

- the energy of $u^{\mathrm{cm}}$ is concentrated at low frequencies (below $0.1 \mathrm{~Hz}$ );

- the signal $u^{\mathrm{cm}}$ is stationary under mild assumptions, as proved in Lemma A1.

This suggests that a random walk model could be sufficient to represent the relevant lowfrequency behavior of $u^{\mathrm{cm}}$

$$
u^{\mathrm{cm}}(k+1)=u^{\mathrm{cm}}(k)+w(k)
$$

where $w$ is a random white Gaussian process with zero-mean and covariance matrix $Q_{w}$. Usually, the covariance matrix $Q_{w}$ is viewed as a design parameter that provides a trade-off between tracking speed and smoothness of the estimates. For simplicity, it is often selected as a diagonal matrix. Faster tracking of the true signals can be obtained by appropriately increasing the elements of $Q_{w}$, which however results in less smooth (i.e. more noisy) estimates and vice versa. A value of $0.1 I_{2}$ is selected for the simulations in this paper. The random walk model is particularly suitable, and often used, for the estimation of an unknown time-varying bias on the state and output equations [25], which has already proved to be an accurate and robust approach to rotor-effective wind speed estimation [17].

Interconnecting the random walk model (20) with the turbine model (19) results in

$$
\begin{aligned}
{\left[\begin{array}{c}
x_{\mathrm{wt}}(k+1) \\
u^{\mathrm{cm}}(k+1)
\end{array}\right] } & =\left[\begin{array}{cc}
A_{\mathrm{wt}} & B_{\mathrm{wt}, u} \\
0 & I
\end{array}\right]\left[\begin{array}{l}
x_{\mathrm{wt}}(k) \\
u^{\mathrm{cm}}(k)
\end{array}\right]+\left[\begin{array}{c}
B_{\mathrm{wt}, \theta} \\
0
\end{array}\right] \theta_{23}^{\mathrm{cm}}(k)+\left[\begin{array}{l}
0 \\
I
\end{array}\right] w(k) \\
y(k) & =\left[\begin{array}{l}
C_{\mathrm{wt}} 0
\end{array}\right]\left[\begin{array}{c}
x_{\mathrm{wt}}(k) \\
u^{\mathrm{cm}}(k)
\end{array}\right]+\left[\begin{array}{c}
D_{\mathrm{wt}, \theta} \\
0
\end{array}\right] \theta_{23}^{\mathrm{cm}}(k)+v(k)
\end{aligned}
$$

A Kalman filter is used to estimate the state of this augmented system. The state estimate $\hat{x}_{\mathrm{wt}}(k)$ will then be used by the LQR controller, discussed next, while the wind signal estimate $\hat{u}_{\mathrm{cm}}(k)$ will be used by the feedforward controller, discussed later on.

The conditions under which system (21) is observable are discussed in [17]. A sufficient condition for the observability is that the pair $\left(A_{\mathrm{wt}}, C_{\mathrm{wt}}\right)$ is observable, and that the turbine system (19) has no poles and zeros at $z=1$, which holds for the models considered in this paper. Note, however, that the augmented system is not controllable, due to the fact that part of the states belong to the wind model. It is assumed that the remaining part of the augmented states (i.e. the pair $\left.\left(A_{\mathrm{wt}}, B_{\mathrm{wt}}\right)\right)$ is controllable.

Given the Kalman filter turbine state estimate, $\hat{x}_{\mathrm{wt}}(k)$, the LQR control action has the form $\left(K_{\text {lqr }} \hat{x}_{\mathrm{wt}}(k)\right)$, where the gain $K_{\text {lqr }}$ is chosen to optimize the following standard quadratic criterion:

$$
J_{\mathrm{lqr}}=\sum_{k=0}^{\infty}\left[\begin{array}{c}
x_{\mathrm{wt}}(k) \\
\theta_{\mathrm{cm}}
\end{array}\right]^{\mathrm{T}}\left[\begin{array}{ll}
Q & \\
& R
\end{array}\right]\left[\begin{array}{c}
x_{\mathrm{wt}}(k) \\
\theta_{\mathrm{cm}}
\end{array}\right]
$$


3.2.2. Feedforward estimated wind disturbance rejection. An additional IPC action is added to the optimal LQG control action, discussed above. It uses a feedforward disturbance rejection control algorithm based on the estimated multi-blade wind speed signal $\hat{u}_{23}^{\mathrm{cm}}(k)$. To this end, define the following transfer functions:

$$
\begin{aligned}
& G(z)=C_{\mathrm{wt}}\left(z I-A_{\mathrm{wt}}\right)^{-1} B_{\mathrm{wt}, \theta}+D_{\mathrm{wt}, \theta} \\
& H(z)=C_{\mathrm{wt}}\left(z I-A_{\mathrm{wt}}\right)^{-1} B_{\mathrm{wt}, u}
\end{aligned}
$$

so that (with slight abuse of notation) it can be expressed $y(k)=G(z) \theta_{23}^{\mathrm{cm}}(k)+H(z) u^{\mathrm{cm}}(k)+v(k)$. Under the assumption of unbiasedness of the Kalman filter estimates, it follows that

$$
y(k)=G(z) \theta_{23}^{\mathrm{cm}}(k)+H(z) \hat{u}^{\mathrm{cm}}(k)+\tilde{v}(k)
$$

where $\tilde{v}(k)$ is a zero-mean random process. The feedforward controller $C_{\mathrm{ff}}(z)$ should hence be designed in such a manner that the control action $\theta_{23}^{\mathrm{cm}}(k)=C_{\mathrm{ff}} G(z) \hat{u}_{23}^{\mathrm{cm}}(k)$ minimizes the influence of $\hat{u}^{\mathrm{cm}}(k)$ on $y(k)$. The optimal controller will then be given by

$$
C_{\mathrm{ff}}(z)=-H(z) G^{-1}(z)
$$

However, it can happen (as is the case with the numerical models considered in this paper) that the transfer function $G(z)$ is a non-minimum phase, resulting in an unstable optimal feedforward controller. To avoid that the inverse of $G(z)$ will be substituted by a stable inverse, as obtained using the SDMI method in [21].

The basic idea behind the SDMI method is, similar to the wind estimation method above, the use of a random walk model. To summarize the method, suppose that $n(k)$ is some (unknown) signal and let $q(k)=G(z) n(k)$. Then finding a stable inverse means computing a stable transfer function $G_{\text {inv }}(z)$ such that $\hat{n}(k)=G_{\text {inv }}(z) q(k) \approx n(k)$. To this end, the signal $n(k)$ can be viewed as an unknown bias that can be estimated using the same idea as the random walk model in combination with a Kalman filter. Expressing $n(k+1)=n(k)+\eta(k)$, with $E\left\{\eta_{k}\right\}=0$ and $E\left\{\eta(k) \eta(k)^{\mathrm{T}}\right\}=Q_{\eta}$, adding this to the state of $G(z)$ and including (if necessary for numerical reasons) a small additional process and/or measurement noise terms results in an augmented model $G_{\text {aug }}(z)$, for which a Kalman filter can be designed. The Kalman filter transfer function from $q(z)$ to $\hat{n}(k)$ represents the inverse of $G(z)$. Hence, for the considered model (22), the SDMI method results in the following stable inverse of $G(z)$ :

$$
G_{\mathrm{inv}}=[0, I]\left(z I-\left[\begin{array}{cc}
A_{\mathrm{wt}} & B_{\mathrm{wt}, \theta} \\
0 & I
\end{array}\right]+K\left[C_{\mathrm{wt}}, 0\right]\right)^{-1} K
$$

The feedforward controller then takes the form $C_{\mathrm{ff}}(z)=-H(z) G_{\mathrm{inv}}(z)$, so that the complete feedback-feedforward control action is formed as shown in Figure 4. This IPC loop, together with the basic power control loop, including the generator torque P-controller and the collective pitch PI-controller, is depicted in Figure 5. The figure shows both the conventional collective pitch control and generator torque control loops, acting on the rotor speed $\Omega$, as well as the IPC algorithm, acting on the measured flapwise blade root bending moments $M_{z i}$ and computing additional actions to the collective pitch angles. Note the required modulation and demodulation of the signals discussed above. 


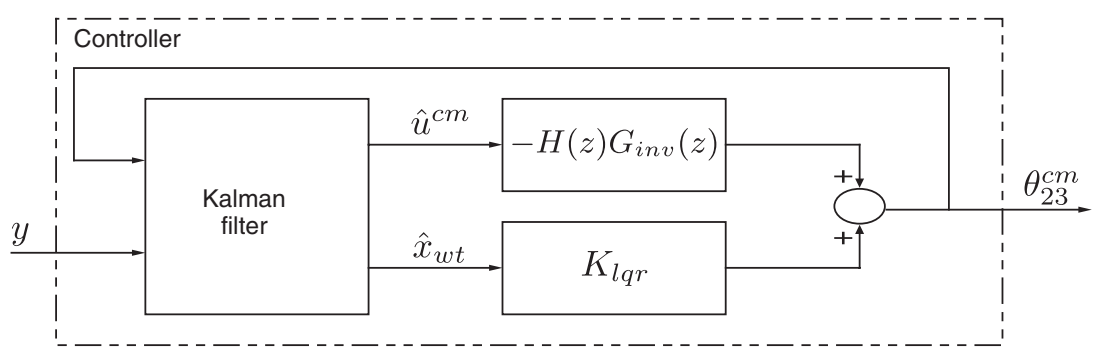

Figure 4. The complete feedback-feedforward control scheme.

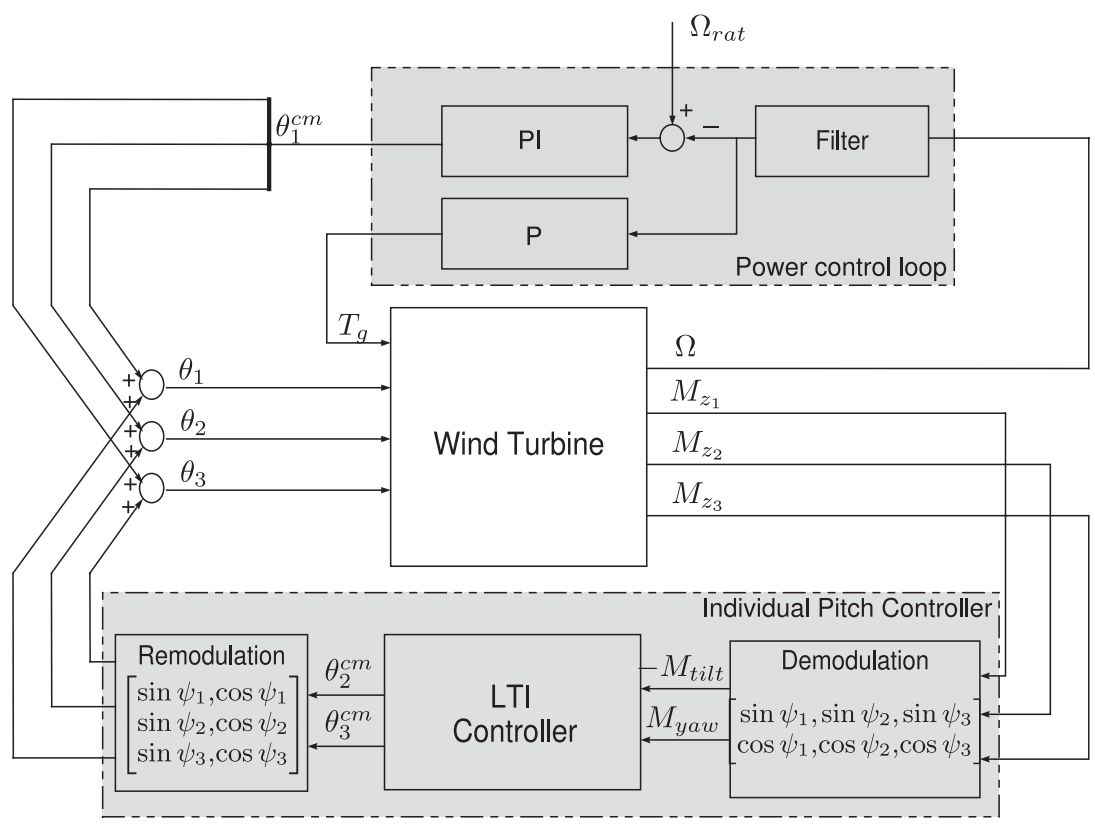

Figure 5. Layout of the complete control structure.

\section{FREQUENCY DOMAIN ANALYSIS}

This section presents results that demonstrate a significant improvement in the load reduction of the non-rotating turbine components, obtained with the presented feedback-feedforward IPC, compared with the conventional PI-based IPC. The controllers are designed and compared on both the simple rigid model and the detailed multi-body TURBU model, as described in Section 2. The parameters of the simple model (12)-(18) for a fictitious $2.5 \mathrm{MW}$ wind turbine are listed in Table I. This model, as well as the TURBU model, is derived for wind speed of $16 \mathrm{~m} / \mathrm{s}$, pitch angle of $10^{\circ}$ and rotor speed of $1.806 \mathrm{rad} / \mathrm{s}$.

The basic speed and power control loops are the same for both IPCs. These are designed as discussed in Section 3.1. The conventional IPC consists of two integrators, one for the tilt-oriented 
Table I. Numerical values of the model parameters in the simple model (12)-(18).

\begin{tabular}{lccc}
\hline Parameter & Value & Parameter & Value \\
\hline$H$ & $55.953 \mathrm{~m}$ & $h_{M_{x}}$ & $8.3806 \times 10^{4} \mathrm{Ns}$ \\
$R_{\mathrm{b}}$ & $40 \mathrm{~m}$ & $h_{F_{z}}$ & $4.0683 \times 10^{3} \mathrm{Ns} / \mathrm{m}$ \\
$J$ & $11.2553 \times 10^{6} \mathrm{~kg} \mathrm{~m}^{2}$ & $h_{M_{z}}$ & $-1.8948 \times 10^{5} \mathrm{~N} \mathrm{~s}$ \\
$m_{\mathrm{tw}}$ & $1.5657 \times 10^{5} \mathrm{~kg}$ & $k_{F_{x}}$ & $-6.1478 \times 10^{3} \mathrm{~N}$ \\
$s_{\mathrm{tw}}$ & $1.235 \times 10^{6} \mathrm{~N} / \mathrm{m}$ & $k_{M_{x}}$ & $-3.7711 \times 10^{4} \mathrm{Nm}$ \\
$d_{\mathrm{tw}}$ & $2.7995 \times 10^{3} \mathrm{~N} \mathrm{~s} / \mathrm{m}$ & $k_{F_{z}}$ & $-1.8306 \times 10^{3} \mathrm{~N}$ \\
$h_{F_{x}}$ & $7.2019 \times 10^{3} \mathrm{Ns} / \mathrm{m}$ & $k_{M_{z}}$ & $1.6174 \times 10^{5} \mathrm{Nm}$ \\
\hline
\end{tabular}

Table II. Parameters used for the design of the feedback-feedforward IPC controller.

\begin{tabular}{lccccc}
\hline Parameter & $Q_{v}$ & $Q_{w}$ & $Q$ & $R$ & $Q_{\eta}$ \\
Value & $0.01 I$ & $0.1 I_{2}$ & $I$ & $0.8 I$ & 0.01 \\
\hline
\end{tabular}

and one for the yaw-oriented channels. The new feedback-feedforward IPC controller is designed as discussed in Section 3.2. The parameters used in the design process are summarized in Table II.

The comparison between the two control designs is performed in the frequency domain. The frequency response plot for the simplified model is depicted in Figure 6. The figure shows the transfer functions from the multi-blade wind signals $u_{23}^{\mathrm{cm}}$ to the rotor tilt and yaw moment $M_{\text {tilt }}$ (left plot) and $M_{\text {yaw }}$ (right plot). The solid lines in the plots represent the case of no IPC, the dashed dotted lines correspond to the conventional IPC method, whereas the dashed lines are for the new feedback-feedforward method. Only the diagonal channels of the transfer function from $u^{\mathrm{cm}}$ to $y$ are given, since the off-diagonal ones are zero (even with the new MIMO controller, which preserves the intrinsic diagonal structure of the simple model). It can be clearly seen from the figure that the conventional IPC approach (dashed line) has good load reduction only at very low frequencies, whereas at $1 \mathrm{p}$ and higher frequencies in the fixed reference frame there is no reduction, and even a slight increase. The low-frequency reduction is due to the integrator structure of this control method, making the method suitable for blade load reduction (as $0 \mathrm{p}$ reduction in $M_{\mathrm{tilt}}$ and $M_{\text {yaw }}$ corresponds to $1 \mathrm{p}$ reduction in the flapwise blade root bending moments), but cannot achieve fatigue-relevant load reduction on the non-rotating components of the wind turbine. On the other hand, by trading off low-frequency load reduction, the proposed feedback-feedforward method achieves reduction over a much wider frequency range, including the $3 p$ frequency, which is very relevant for fatigue on the non-rotating components, such as the nacelle, yaw bearing and tower [16]. Improved reduction at low frequencies can be obtained by including integral action in the controller.

Similar conclusions can be made based on the results with the TURBU model, as depicted in Figure 7. Now there is coupling between tilt- and yaw-oriented moments, so that the offdiagonal channels are also plotted. Note that although the IPC controller design has been performed based on the reduced lumped TURBU model, the results in the figure represent the closed-loop system with the detailed multi-body TURBU model. Note also that the advanced feedbackfeedforward controller achieves improved load reduction over a much wider frequency band than the conventional IPC method, which only leads to improvement at very low frequencies, whereas it actually results in load amplification at frequencies of $1 \mathrm{p}$ (here $0.3 \mathrm{~Hz}$ ) and higher in the fixed 

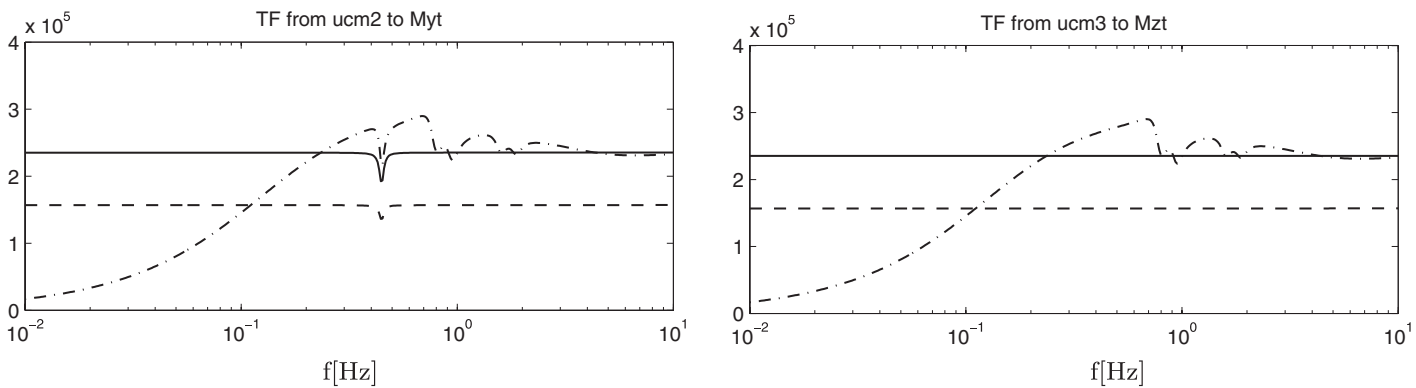

Figure 6. Frequency response plot of tilt moment $M_{\text {tilt }}$ (left) and yaw moment $M_{\text {yaw }}$ (right) due to the multi-blade wind inputs $u_{2}^{\mathrm{cm}}$ and $u_{3}^{\mathrm{cm}}$, respectively, for the simple rigid turbine model with no IPC (solid), conventional IPC (dashed dotted) and new feedback-feedforward IPC (dashed).


Figure 7. Frequency response plot of tilt moment $M_{\text {tilt }}$ (first row) and yaw moment $M_{\text {yaw }}$ (second row) due to the multi-blade wind inputs $u_{2}^{\mathrm{cm}}$ (left column) and $u_{3}^{\mathrm{cm}}$ (right column) for the TURBU model with no IPC (solid), conventional IPC (dashed dotted) and new feedback-feedforward IPC (dashed).

reference frame (observe the high peak on the top-left plot in Figure 7, which is at about 1p). The performance of the present method at low frequencies can easily be improved by including integral action in the controller. Finally, it needs to be mentioned that the improved high-frequency reduction inevitably requires pitch control activity at these frequencies, which might in practice be undesirable. This can be circumvented by introducing additional penalty on the control signal at high frequencies in the optimal control optimization. 


\section{CONCLUSION}

This paper presented a new approach to the design of IPC controllers for load reduction. The method is based on a two-degree-of-freedom control structure, consisting of an optimal feedback LQG controller and an additional feedforward disturbance rejection controller based on estimated wind signals. The controller design is performed based on the wind turbine with fictitious inputs and outputs that are uniquely defined from blade pitch angles and the measured blade flapwise root bending moments. These fictitious multi-blade inputs and outputs are computed using the Coleman transformation and lead to a purely time-invariant wind turbine model. This approach, contrary to the one followed in [18] where the design is performed directly on the periodic system, makes it possible to exploit the very well-developed control design theory for linear systems, and allows for extension to the case of varying operating conditions (wind speed, rotor speed and pitch angle), as suggested for collective pitch control in the work of Bianchi et al. [7]. The wind estimation is based on a simple but effective random walk model, avoiding the need for an accurate wind model. The new IPC design method is tested on two linearized turbine models of different complexities, and the results are compared with those obtained with the conventional IPC control.

\section{APPENDIX A}

Lemma A1 (Stationarity of multi-blade wind speeds)

Under the assumption of homogeneous turbulence, constant rotational speed and non-oblique oriented wind flow, the multi-blade components $u_{i}^{\mathrm{cm}}, i=1,2,3$, of the blade-effective wind speeds are stationary processes.

\section{Proof}

For homogeneous turbulence and purely axial wind direction, the blade-effective wind speed $u_{i}$ on a fixed point on a rotating blade can be expressed as a time-varying Fourier expansion

$$
u_{i}\left(t, \psi_{i}\right)=\sum_{p=-\infty}^{\infty} \mathrm{e}^{\mathrm{j} p \psi_{i}(t)} \hat{u}_{p}(t), \quad \hat{u}_{p}(t)=\frac{1}{2 \pi} \int_{0}^{2 \pi} \mathrm{e}^{\mathrm{j} p \phi} u(t, \phi) \mathrm{d} \phi
$$

where $\hat{u}_{p}(t)$ are time-dependent rotational modes. It has been shown in [12] that the following expression holds for the multi-blade coordinates of the blade-effective wind speeds:

$$
\left[\begin{array}{l}
u_{1}^{\mathrm{cm}}(t) \\
u_{2}^{\mathrm{cm}}(t) \\
u_{3}^{\mathrm{cm}}(t)
\end{array}\right]=\sum_{m=-\infty}^{\infty} \mathrm{e}^{\mathrm{j} 3 m \psi}\left[\begin{array}{c}
\hat{u}_{3 m}(t) \\
\mathrm{j}\left(\hat{u}_{3 m+1}(t)-\hat{u}_{3 m-1}(t)\right) \\
\mathrm{j}\left(\hat{u}_{3 m+1}(t)+\hat{u}_{3 m-1}(t)\right)
\end{array}\right]
$$

Then, with $a^{*}$ denoting the conjugate of $a$, it can easily be shown that

$$
\left[\begin{array}{l}
u_{1}^{\mathrm{cm}}(t+\tau) \\
u_{2}^{\mathrm{cm}}(t+\tau) \\
u_{3}^{\mathrm{cm}}(t+\tau)
\end{array}\right]^{*}=\sum_{n=-\infty}^{\infty} \mathrm{e}^{-\mathrm{j} 3 n \psi}\left[\begin{array}{c}
\hat{u}_{3 n}(t+\tau) \\
-\mathrm{j}\left(\hat{u}_{3 n+1}^{*}(t+\tau)-\hat{u}_{3 n-1}^{*}(t+\tau)\right) \\
-\mathrm{j}\left(\hat{u}_{3 n+1}^{*}(t+\tau)+\hat{u}_{3 n-1}^{*}(t+\tau)\right)
\end{array}\right]
$$


Therefore, for the variance of $u_{2}^{\mathrm{cm}}$ one has

$$
\begin{aligned}
E & \left\{u_{2}^{\mathrm{cm}}(t)\left(u_{2}^{\mathrm{cm}}(t+\tau)\right)^{*}\right\} \\
& =E\left\{\sum_{m, n=-\infty}^{\infty} \mathrm{e}^{\mathrm{j} 3(m-n) \psi}\left(\hat{u}_{3 m+1}(t)-\hat{u}_{3 m-1}(t)\right)\left(\hat{u}_{3 n+1}^{*}(t+\tau)-\hat{u}_{3 n-1}^{*}(t+\tau)\right)\right\} \\
& =\sum_{m, n=-\infty}^{\infty} \mathrm{e}^{\mathrm{j} 3(m-n) \psi} E\left\{\left(\hat{u}_{3 m+1}(t)-\hat{u}_{3 m-1}(t)\right)\left(\hat{u}_{3 n+1}^{*}(t+\tau)-\hat{u}_{3 n-1}^{*}(t+\tau)\right)\right\}
\end{aligned}
$$

since under the assumption of constant rotational speed one has $\psi=\Omega t+\psi(0)$, so that $\mathrm{e}^{\mathrm{j} 3(m-n) \psi}$ are purely deterministic signals. Furthermore, in [26] it is proved that, under the considered assumptions, the rotational modes are orthogonal and stationary, i.e.

$$
\left\{\hat{u}_{p}(t) \hat{u}_{q}^{*}(t+\tau)\right\}=\delta_{p, q} \sigma_{\hat{u}_{p}}(\tau)
$$

where $\delta_{p, q}$ denotes the Kronecker delta function and $\sigma_{\hat{u}_{p}}(\tau)$ is the covariance function of $\hat{u}_{p}$. Therefore, in the above expression for $E\left\{u_{2}^{\mathrm{cm}}(t)\left(u_{2}^{\mathrm{cm}}(t+\tau)\right)^{*}\right\}$ all terms for $n \neq m$ drop, giving

$$
E\left\{u_{2}^{\mathrm{cm}}(t)\left(u_{2}^{\mathrm{cm}}(t+\tau)\right)^{*}\right\}=\sum_{m=-\infty}^{\infty}\left(\sigma_{\hat{u}_{3 m-1}}(\tau)+\sigma_{\hat{u}_{3 m+1}}(\tau)\right)
$$

Clearly, the correlation function of $u_{2}^{\mathrm{cm}}(t)$ is not a function of the time $t$. The same lines can be followed for the first and the third multi-blade components $u_{1}^{\mathrm{cm}}(t)$, and $u_{3}^{\mathrm{cm}}(t)$ to arrive at the same conclusion. Therefore, $u_{i}^{\mathrm{cm}}(t), i=1,2,3$, are stationary processes.

\section{REFERENCES}

1. Bossanyi E. The design of closed loop controllers for wind turbines. Wind Energy 2000; 3:149-163.

2. Burton T, Sharpe D, Jenkins N, Bossanyi E. Handbook of Wind Energy. Wiley: New York, 2001.

3. van der Hooft E, van Engelen T, Schaak P. Development of wind turbine control algorithm for industrial use. Proceedings of the European Wind Energy Conference, Copenhagen, Denmark, 2001.

4. van Engelen T, Markou H, Buhl T, Marrant B. Morphological study for aeroelastic control concepts for wind turbines. Technical Report ECN-E-06-056, Energy Research Center for the Netherlands (ECN), 2006 (available from http://www.ecn.nl/publicaties/default.aspx?nr=ECN-E-06-056).

5. Geyler M, Caselitz P. Individual blade pitch control design for load reduction on large wind turbines. Proceedings of the European Wind Energy Conference, Milan, Italy, 2007; 82-86.

6. Cutululis N, Ceanga E, Hansen A, Sørensen P. Robust multi-model control of an autonomous wind power system. Wind Energy 2006; 9:399-419.

7. Bianchi F, De Battista H, Mantz R. Wind Turbine Control Systems: Principles, Modelling and Gain-scheduling Design (Advances in Industrial Control). Springer: London, 2006.

8. Lescher F, Zhao J, Borne P. Robust gain scheduling controller for pitch regulated variable speed wind turbine. Studies in Informatics and Control 2005; 14(4):299-315.

9. Bianchi F, Mantz R, Christiansen C. Control of variable-speed wind turbines by LPV gain scheduling. Wind Energy 2004; 7:1-8.

10. Bianchi F, Mantz R, Christiansen C. Gain scheduling control of variable-speed wind energy conversion systems using quasi-LPV models. Control Engineering Practice 2005; 13:247-255.

11. Lescher F, Camblong H, Curea O, Briand R. LPV control of wind turbines for fatigue loads reduction using intelligent micro sensors. Proceedings of the 2007 American Control Conference, New York City, U.S.A., 2007; 6061-6066. 
12. van Engelen T. Design model and load reduction assessment for multi-rotational mode individual pitch control (higher harmonics control). Proceedings of the European Wind Energy Conference, Athens, Greece, 2006 (available from: http://www.ecn.nl/publicaties/default.aspx?nr=ECN-RX-06-068).

13. Bossanyi E. Individual blade pitch control for load reduction. Wind Energy 2003; 6:1919-128.

14. Bossanyi E. Wind turbine control for load reduction. Wind Energy 2003; 6:229-244.

15. Kallesœ BS. A low-order model for analysing effects of blade fatigue load control. Wind Energy 2006; 9:421-436.

16. Bossanyi E. Further load reductions with individual pitch control. Wind Energy 2005; 8:481-485.

17. Mesic S, Verdult V, Verhaegen M, Kanev S. Estimation and robustness analysis of actuator faults based on Kalman filtering. Proceedings of the 5th Symposium on Fault Detection, Supervision and Safety for Technical Processes, Washington, DC, U.S.A., 2003.

18. Stol K, Balas M. Periodic disturbance accommodating control for blade load mitigation in wind turbines. Journal of Solar Energy Engineering 2003; 125(4):379-385.

19. Kendall L, Balas M, Lee Y, Fingersh L. Application of proportional-integral and disturbance accommodating control to variable speed variable pitch horizontal axis wind turbines. Wind Engineering 1997; 21:21-38.

20. Goodwin G, Graebe S, Salgado M. Control System Design. Prentice-Hall: Englewood Cliffs, NJ, 2001.

21. George K. Verhaegen M, Scherpen J. A systematic and numerically efficient procedure for stable dynamic model inversion of LTI systems. Proceedings of the 38th Conference on Decision and Control, Pheonix, AZ, U.S.A., 1999; 1881-1886.

22. van Engelen T. Control design based on aero-hydro-servo-elastic linear models from TURBU (ECN). Proceedings of the European Wind Energy Conference, Milan, Italy, 2007.

23. Coleman R, Feingold A. Theory of Self-excited Mechanical Oscillations of Helicopter Rotors with Hinged Blades. USGPO: Washington, DC, 1958.

24. Hansen M, Thomsen K, Fuglsang P, Knudsen T. Two methods for estimating aeroelastic damping of operational wind turbine modes from experiments. Wind Energy 2006; 9:179-191.

25. Kanev S, Verhaegen M. Two-stage Kalman filtering via structured square-root. Journal of Communications in Informations and Systems 2005; 5(1):143-168.

26. van Engelen T, Schaak P. Oblique inflow model for assessing wind turbine controllers. Proceedings of the 2nd Conference on the Science of Making Torque from Wind, Denmark, 2007. 